Article

\title{
Psychometric Validation of the German Translation of the Quality of Life Questionnaire-Bronchiectasis (QOL-B)-Data from the German Bronchiectasis Registry PROGNOSIS
}

\author{
Laura Quellhorst ${ }^{1}$, Grit Barten-Neiner ${ }^{2,3}$, Andrés de Roux ${ }^{4}$, Roland Diel ${ }^{5,6,7}$, Pontus Mertsch ${ }^{8}$, Isabell Pink ${ }^{1}$, \\ Jessica Rademacher 1,3, Sivagurunathan Sutharsan ${ }^{9}$, Tobias Welte 1,3, Annegret Zurawski 1,3, \\ Felix C. Ringshausen $1,3, * \mathbb{D}$ and on behalf of the PROGNOSIS study group ${ }^{\dagger}$
}

check for updates

Citation: Quellhorst, L.;

Barten-Neiner, G.; de Roux, A.; Diel,

R.; Mertsch, P.; Pink, I.; Rademacher,

J.; Sutharsan, S.; Welte, T.; Zurawski,

A.; et al. Psychometric Validation of the German Translation of the Quality of Life QuestionnaireBronchiectasis (QOL-B) - Data from the German Bronchiectasis Registry PROGNOSIS. J. Clin. Med. 2022, 11, 441. https://doi.org/10.3390/ jcm11020441

Academic Editor: Anne Chang

Received: 5 December 2021

Accepted: 13 January 2022

Published: 15 January 2022

Publisher's Note: MDPI stays neutral with regard to jurisdictional claims in published maps and institutional affiliations.

Copyright: (C) 2022 by the authors. Licensee MDPI, Basel, Switzerland. This article is an open access article distributed under the terms and conditions of the Creative Commons Attribution (CC BY) license (https:// creativecommons.org/licenses/by/ $4.0 /)$.
1 Department of Respiratory Medicine, Hannover Medical School (MHH), 30625 Hannover, Germany; LauraQuellhorst@gmx.de (L.Q.); Pink.Isabell@mh-hannover.de (I.P.); Rademacher.Jessica@mh-hannover.de (J.R.); Welte.Tobias@mh-hannover.de (T.W.); Zurawski.Annegret@mh-hannover.de (A.Z.)

2 CAPNETZ STIFTUNG, Hannover Medical School (MHH), 30625 Hannover, Germany; Barten.Grit@mh-hannover.de

3 Biomedical Research in End-Stage and Obstructive Lung Disease Hannover (BREATH), German Center for Lung Research (DZL), 30625 Hannover, Germany

4 Pneumologische Praxis am Schloss Charlottenburg, 14059 Berlin, Germany; drderoux.a@gmail.com

5 Institute for Epidemiology, University Medical Center Schleswig-Holstein, 24105 Kiel, Germany; roland.diel@epi.uni-kiel.de

6 LungenClinic Grosshansdorf, Airway Research Center North (ARCN), German Center for Lung Research (DZL), 22927 Grosshansdorf, Germany

7 Institution for Statutory Accident Insurance and Prevention in the Health and Welfare Services (BGW) 22089 Hamburg, Germany

8 Department of Medicine V, University Hospital, LMU Munich, Comprehensive Pneumology Center Munich (CPC-M), German Center for Lung Research (DZL), 81377 Munich, Germany:

Pontus.Mertsch@med.uni-muenchen.de

9 Department of Pulmonary Medicine, University Hospital Essen, Ruhrlandklinik, University Duisburg-Essen, 45239 Essen, Germany; Sivagurunathan.Sutharsan@rlk.uk-essen.de

* Correspondence: Ringshausen.Felix@mh-hannover.de; Tel.: +49-511-532-3595

+ The PROGNOSIS study group Members are listed in acknowledgments.

\begin{abstract}
Patients with bronchiectasis feature considerable symptom burden and reduced healthrelated quality of life (QOL). We provide the psychometric validation of the German translation of the disease-specific Quality of Life Questionnaire-Bronchiectasis (QOL-B), version 3.1, using baseline data of adults consecutively enrolled into the prospective German bronchiectasis registry PROGNOSIS. Overall, 904 patients with evaluable QOL-B scores were included. We observed no relevant floor or ceiling effects. Internal consistency was good to excellent (Cronbach's $\alpha \geq 0.73$ for each scale). QOL-B scales discriminated between patients based on prior pulmonary exacerbations and hospitalizations, breathlessness, bronchiectasis severity index, lung function, sputum volume, Pseudomonas aeruginosa status and the need for regular pharmacotherapy, except for Social Functioning, Vitality and Emotional Functioning scales. We observed moderate to strong convergence between several measures of disease severity and QOL-B scales, except for Social and Emotional Functioning. Two-week test-retest reliability was good, with intraclass correlation coefficients $\geq 0.84$ for each scale. Minimal clinical important difference ranged between 8.5 for the Respiratory Symptoms and 14.1 points for the Social Functioning scale. Overall, the German translation of the QOL-B, version 3.1, has good validity and test-retest reliability among a nationally representative adult bronchiectasis cohort. However, responsiveness of QOL-B scales require further investigation during registry follow-up.
\end{abstract}

Keywords: bronchiectasis; Germany; patient-reported outcome measures; quality of life; questionnaire design; registries 


\section{Introduction}

Bronchiectasis is a chronic suppurative and often progressive airway disease, which manifests with considerable symptom burden, in particular persistent productive cough, reduced exercise capacity, frequent pulmonary exacerbations and reduced health-related quality of life (QOL) [1]. Its prevalence has been increasing over the past years in many settings, resulting in substantial economic burden to healthcare systems [2-4].

In randomized controlled trials (RCTs) in bronchiectasis, various objective measures such as sputum bacterial load or pulmonary exacerbations, but also patient-reported outcome measures (PROMs) such as QOL have been used to evaluate the efficacy of different therapeutic interventions [5-8]. In this regard, PROMs are not only important as a patient priority [9], but have gained importance for regulatory agencies as trial endpoints [10,11].

So far, a variety of different tools have been applied to assess QOL in bronchiectasis populations, either using well-established respiratory health-related QOL questionnaires such as the St. George's Respiratory Questionnaire (SGRQ) or disease-specific QOL questionnaires such as the Quality of Life Questionnaire-Bronchiectasis (QOL-B), which is the first tool specifically developed for use in bronchiectasis [8,12-15]. To validate those questionnaires accepted measures of symptom burden and disease severity have been used, including breathlessness, sputum volume, forced expiratory volume in one second $\left(\mathrm{FEV}_{1}\right)$, prior pulmonary exacerbations and hospitalizations, infection status and radiology [16,17].

While the disease-specific QOL-B has been validated in an international multi-center RCT as well as a Spanish bronchiectasis population [12,18], its correlates with symptom burden and disease severity among German adults with bronchiectasis are unknown.

In order to investigate QOL among this population, the Prospective German Non-CF Bronchiectasis Patient Registry (PROGNOSIS) applies the respective translation of the QOL-B, version 3.1, at baseline visits and during annual follow-up [19], thus offering an opportunity to explore its validity in a real-world application. Therefore, the aim of our study was to provide a psychometric validation of the German translation of the QOL$\mathrm{B}$, version 3.1, using baseline data of the first 1000 patients consecutively enrolled into PROGNOSIS.

\section{Materials and Methods}

\subsection{Database}

In summary, PROGNOSIS (www.bronchiektasen-register.de, accessed on 12 January 2022; registered at Clinicaltrials.gov under the identifier NCT02574143; hosted at Hannover Medical School [MHH], Hannover, Germany) is a currently ongoing, prospective and non-interventional registry, which was launched in June 2015 with the initial aim to recruit 750 patients with computed tomography (CT)-confirmed bronchiectasis from at least 10 sites across Germany over 3 consecutive years [19]. Since then, $>1500$ patients have been enrolled from a total of 38 sites across all levels of healthcare, including 15 respiratory physicians in private practice as well as 13 teaching and 10 university hospitals due to expanded funding, an associated partnership with the German Center for Lung Research (DZL, Giessen, Germany) and the continuous support of CAPNETZ STIFTUNG (www.capnetz.de, accessed 12 January 2022). This ensured broad geographic and epidemiological representativeness, with about half of patients enrolled at university hospitals and one quarter at private practices and teaching hospitals, each. PROGNOSIS prospectively collects baseline, annual ( \pm 3 months) follow-up and outcome data, using a standardized electronic case report form (eCRF) via an online database accessible through the registry's homepage. Pseudonymization and electronic storage of clinical data follow a uniform and quality-assured standard operating procedure according to German and European Data Protection Act. The PROGNOSIS protocol and eCRF have been harmonized with those of the European bronchiectasis registry EMBARC (www.bronchiectasis.eu, accessed 12 January 2022) in order to allow data sharing and joint data analyses. We recorded comprehensive clinical data including not only demographics, disease history, etiological testing, complications and comorbidities, microbiology, pulmonary function, radiology 
and treatment, but also measures of disease severity and symptom burden. The multidimensional Bronchiectasis Severity Index (BSI) was calculated as previously described [17]. Pulmonary exacerbations were defined as the need for a significant change in medical management for acute respiratory symptoms, typically requiring antibiotics, and recorded from either patient history, hospital and/or prescription records or a combination of those. Etiology was recorded by the site investigators and verified centrally using the patients etiological testing data. Chronic infection was defined as two isolates of the same pathogen at least 3 months apart over 1 year while in a stable state [1]. Spirometry was performed according to American Thoracic Society/European Respiratory Society standards and predicted values were calculated centrally using Global Lung Function Initiative equations. Radiological severity was assessed locally by the site investigators in the patients' most recent CT scans according to the modified Reiff score [20]. All datasets were externally validated and, thereafter, manually cleaned by the authors before analysis. PROGNOSIS received ethical approval by the ethic committees of all participating centers, referring to the initial ethical approval of MHH's institutional review board (No. 6656/2015).

\subsection{Patient Population and Study Design}

Inclusion criteria are age $\geq 18$ years, CT-confirmed bronchiectasis and prior written and informed consent, while known cystic fibrosis at the time of inclusion and prior lung/heartlung transplantation exclude patients from participation. For the present analysis, only patients were selected in whom at least one baseline QOL-B scale was evaluable based on responses to the respective items. We conducted a cross-sectional analysis of baseline data with the aim to provide the psychometric validation of the German translation of the QOL-B, version 3.1, though excluding the evaluation of responsiveness over time.

\subsection{Quality of Life Assessment}

Disease-specific QOL was determined at the baseline visit by means of the German translation of the QOL-B, version 3.1, for which prior written permission of the copyright holder had been obtained [12,21]. However, completion of QOL-B was optional. Patients were instructed to answer the QOL-B on their own (self-administered), while at the hospital or during a clinic or private practice visit. The QOL-B consists of 37 items that subdivide into 8 scales (Respiratory Symptoms, Physical Functioning, Vitality, Role Functioning, Health Perceptions, Emotional Functioning, Social Functioning and Treatment Burden), with scores ranging from 0 to 100 within each scale. No total score is calculated. Patients not receiving bronchiectasis treatment were instructed to skip the Treatment Burden scale. All items were scored using the SAS and SPSS Program Codes for Scoring the QOL-B Version 3.1 , according to the copyright holder's instructions, and rechecked manually by random sampling (Supplemental file S1) [12,21]. If responses were missing for more than half the items in a scale, the score for that scale was not calculated. Missing QOL-B values were not imputed (Supplemental file S1). Discriminant and convergent validity of QOL-B scales were assessed using pulmonary exacerbations, hospitalizations, breathlessness (Medical Research Council [MRC] dyspnea scale), BSI, $\mathrm{FEV}_{1}$, patient-estimated average daily sputum volume, Pseudomonas aeruginosa infection status, need for regular pharmacological treatment, radiological severity and prior thoracic surgery as markers of bronchiectasis severity and symptom burden.

\subsection{Statistical Analysis}

Continuous data are presented as mean with standard deviation (SD) or median with interquartile range according to their distribution, categorical data as numbers and percentages. The Kolmogorov-Smirnov test was used to assess distribution of continuous data. Floor and ceiling effects were assessed by descriptive statistics. We assumed floor and ceiling effects to be relevant, if $\geq 15 \%$ of respondents had scores of 0 and 100 within a scale, respectively [12,16]. Internal consistency was evaluated using Cronbach's $\alpha$. Short-term test-retest reliability (reproducibility) was estimated with intraclass correlation coefficients 
(ICCs) in a subgroup of 20 randomly selected patients at MHH's adult bronchiectasis clinic in the absence of a change in clinical status over a $14( \pm 7)$-day period. The Mann-Whitney U and the Kruskal-Wallis test were used to assess differences between groups in the analysis of discriminant validity, while Spearman's correlations were calculated to assess convergent validity of QOL-B scales. Rho $(r)$ values $<0.3$ indicate weak, values of 0.3 to 0.49 moderate and values $>0.5$ strong correlation $[15,22]$. We considered a two-sided $p$-value $<0.05$ statistically significant. Minimal clinical important difference (MCID) estimates were assessed distribution-based by the standard error of the mean method (SEM $=\mathrm{SD} \sqrt{ }(1-\alpha))$. Overall, missing values were infrequently observed in spirometry $\left(\mathrm{FEV}_{1} 6.4 \%\right)$, breathlessness (MRC dyspnea scale $5.8 \%$ ), number of pulmonary exacerbations in the previous 12 months (2.6\%) and body mass index $(0.2 \%)$. In order to utilize all datasets with evaluable QOL-B scales we calculated missing values by the multiple imputation method, with age, sex, comorbidities, etiology, prior hospitalizations, Pseudomonas aeruginosa infection status and radiological severity as predictor variables. The number of imputations was set to 10. All analyses were performed with SPSS, version 27.0 (IBM Corp., Armonk, NY, USA).

\section{Results}

We consecutively recruited 1000 adults with CT-confirmed bronchiectasis into the PROGNOSIS registry from July 2015 to March 2018. Of those, 904 patients (90.4\%) completed the QOL-B at baseline and had at least one evaluable baseline QOL-B scale, representing the final study population for the present analysis. Table 1 shows the demographic and baseline characteristics, which were comparable between patients who had filled out the QOL-B and those who had not (Supplementary S2 Table S1).

Table 1. Demographic and baseline characteristics $(n=904)$.

\begin{tabular}{lr}
\hline Variable & Value \\
\hline Age (years), mean (SD) & $59.5(15.8)$ \\
\hline Females, $\mathrm{n}(\%)$ & $538(59.5)$ \\
\hline BMI $\left(\mathrm{kg} / \mathrm{m}^{2}\right)$, mean $(\mathrm{SD})$ & $24.1(4.5)$ \\
\hline FEV $1 \%$ predicted, mean (SD) & $69.8(26.8)$ \\
$\geq 80 \%$ predicted, $\mathrm{n}(\%)$ & $344(38.1)$ \\
$50-79 \%$ predicted, $\mathrm{n}(\%)$ & $322(35.6)$ \\
$30-49 \%$ predicted, $\mathrm{n}(\%)$ & $180(19.9)$ \\
$<30 \%$ predicted, $\mathrm{n}(\%)$ & $58(6.4)$ \\
\hline Radiological severity, $\mathrm{n}(\%)$ & $325(36.0)$ \\
$<3$ lobes affected & $579(64.0)$ \\
$\geq 3$ lobes affected and $/$ or cystic & \\
bronchiectasis & $751(83.1)$ \\
\hline MRC dyspnea scale, $\mathrm{n}(\%)$ & $153(16.9)$ \\
$1-3$ & \\
$4-5$ & $57(6.3)$ \\
\hline Smoking, $\mathrm{n}(\%)$ & $335(37.1)$ \\
Active smoker & $512(56.6)$ \\
Former smoker & $1(0-3)$ \\
Never smoked & $271(30.0)$ \\
\hline Exacerbations in the past 12 months, median & $361(39.9)$ \\
(IQR) & $272(30.1)$ \\
$0, \mathrm{n}(\%)$ & \\
$1-2, \mathrm{n}(\%)$ & \\
$\geq 3, \mathrm{n}(\%)$ & \\
\hline & \\
\hline
\end{tabular}


Table 1. Cont.

\begin{tabular}{|c|c|}
\hline Variable & Value \\
\hline Prior hospital admission, $\mathrm{n}(\%)^{1}$ & $349(38.6)$ \\
\hline $\begin{array}{l}\text { Hospitalizations in the past } 12 \text { months, median } \\
\text { (IQR) }{ }^{1}\end{array}$ & $0(0-1)$ \\
\hline $\begin{array}{l}\text { Regular pharmacological treatment of } \\
\text { bronchiectasis, } \mathrm{n}(\%)\end{array}$ & $704(77.9)$ \\
\hline Regular sputum production, $\mathrm{n}(\%)$ & $704(77.9)$ \\
\hline Average daily sputum volume, median (IQR) & $20(10-50)$ \\
\hline $0 \mathrm{~mL} /$ day, $\mathrm{n}(\%)$ & $284(31.4)$ \\
\hline 1-10 mL/day, n (\%) & $261(28.9)$ \\
\hline 11-20 mL/day, n (\%) & $122(13.5)$ \\
\hline 21-50 mL/day, n (\%) & $151(16.7)$ \\
\hline 51-100 mL/day, n (\%) & $60(6.6)$ \\
\hline$>100$ mL/day, n (\%) & $26(2.9)$ \\
\hline \multicolumn{2}{|l|}{ BSI category $(n=666), \mathrm{n}(\%)^{2}$} \\
\hline Mild (0-4) & $150(22.5)$ \\
\hline Moderate (5-8) & $390(58.6)$ \\
\hline Severe $(\geq 9)$ & $126(18.9)$ \\
\hline
\end{tabular}

Patients had a mean (SD) age of 59.5 (15.8) years, ranging from 18 to 93 years, showed a slight female predominance (59.5\%) and, on average, had moderate airflow obstruction on spirometry (mean [SD] $\mathrm{FEV}_{1} 69.8 \%$ [26.8] predicted; mean [SD] Tiffeneau index 0.69 [0.15]; Table 1). The five most common etiologies of bronchiectasis were idiopathic in $337(37.3 \%)$, postinfectious in $180(19.9 \%)$, COPD in $133(14.7 \%)$, asthma in $99(11.0 \%)$ and primary ciliary dyskinesia/Kartagener syndrome in 79 patients (8.7\%). Likewise, the most common comorbidities with a frequency of $>10 \%$ were cardiovascular in $354(39.2 \%)$, chronic rhinosinusitis in $270(29.9 \%)$, asthma and COPD in each $269(29.8 \%)$, gastro-esophageal reflux in $166(18.4 \%)$, nasal polyps in $141(15.6 \%)$, malignancy in $108(11.9 \%)$ and osteoporosis in $98(10.8 \%)$. Depression and anxiety disorders were reported in $86(9.5 \%)$ and 34 patients (3.8\%), respectively (Supplementary S2 Table S1). Pseudomonas aeruginosa was the most common respiratory pathogen in 223 of 680 patients $(32.8 \%)$, of whom sputum culture results were available at baseline, followed by Staphylococcus aureus in $112(16.5 \%)$, Haemophilus influenzae in 93 (13.7\%), Aspergillus fumigatus in 73 (10.7\%) and nontuberculous mycobacteria in 41 patients (6.0\%; Supplementary S2 Table S1).

\subsection{Floor and Ceiling Effects, Internal Consistency and Test-Retest Reliability}

Mean baseline QOL-B scores, the assessment of floor (score $=0$ ) and ceiling effects (score $=100$ ) as well as internal consistency are shown in Table 2. In summary, we observed no floor and ceiling effects in $\geq 15 \%$ of patients. Although $10.7 \%$ of patients had a Physical Functioning score of 0 , we found that $\leq 4.7 \%$ had a score of 0 on any other scale. Similarly, $10.9 \%, 9.1 \%$ and $7.0 \%$ of patients showed an Emotional, Social and Role Functioning score of 100 , respectively, but $\leq 4.7 \%$ had a score of 100 on any other scale (Table 2). Moreover, we evaluated internal consistency using Cronbach's $\alpha$. We found that all values were $\geq 0.73$, thus indicating a coherent and reliable construct with strong correlation among the items on each scale (Table 2). Next, we evaluated test-retest reliability in a subgroup of 20 randomly selected subjects, who repeated the QOL-B in the absence of a change in clinical status within a 14( \pm 7$)$-day interval. Here, we observed ICCs $\geq 0.84$ for each scale, indicating good to excellent reproducibility (Table 2). 
Table 2. QOL-B scores at baseline, floor and ceiling effects, internal consistency and test-retest reliability.

\begin{tabular}{|c|c|c|c|c|c|c|}
\hline QOL-B Scale & $n^{1}$ & $\begin{array}{c}\text { Mean (SD) } \\
\text { QOL-B Scores }\end{array}$ & $\begin{array}{c}\text { Floor Effects, } \\
\text { n (\%) }\end{array}$ & $\begin{array}{c}\text { Ceiling Effects, } \\
\text { n (\%) }\end{array}$ & Cronbach's $\alpha$ & $\begin{array}{c}\text { ICC } \\
(95 \% \text { CI })\end{array}$ \\
\hline Respiratory Symptoms & 892 & $56.2(21.0)$ & $1(0.1)$ & $9(1.0)$ & 0.84 & $0.93(0.82-0.97)$ \\
\hline Physical Functioning & 889 & $41.8(29.8)$ & $95(10.7)$ & $42(4.7)$ & 0.92 & $0.96(0.90-0.99)$ \\
\hline Vitality & 892 & $42.0(21.4)$ & $40(4.5)$ & $10(1.1)$ & 0.76 & $0.94(0.84-0.97)$ \\
\hline Role Functioning & 898 & $58.8(27.4)$ & $23(2.6)$ & $63(7.0)$ & 0.86 & $0.88(0.69-0.95)$ \\
\hline Health Perceptions & 891 & $36.3(22.6)$ & $42(4.7)$ & $3(0.3)$ & 0.79 & $0.84(0.59-0.94)$ \\
\hline Emotional Functioning & 889 & $69.2(21.9)$ & $4(0.4)$ & $97(10.9)$ & 0.82 & $0.94(0.85-0.98)$ \\
\hline Social Functioning & 878 & $59.9(26.9)$ & $31(3.5)$ & $80(9.1)$ & 0.73 & $0.87(0.66-0.95)$ \\
\hline Treatment Burden & 645 & $51.3(25.1)$ & $18(2.8)$ & $27(4.2)$ & 0.73 & $0.90(0.73-0.96)$ \\
\hline
\end{tabular}

${ }^{1}$ If responses were missing for more than half the items in a scale, the score for that scale was not calculated. Patients not receiving bronchiectasis treatment were instructed to skip the Treatment Burden scale. Abbreviations: $\mathrm{CI}$, confidence interval; ICC, intraclass correlation coefficient; QOL-B, Quality of Life Questionnaire-Bronchiectasis; $\mathrm{SD}$, standard deviation.

\subsection{Discriminant and Convergent Validity of QOL-B Scores}

In order to assess discriminant validity, we compared mean baseline QOL-B scores with a variety of measures of disease severity and symptom burden. We found significant discrimination between subjects by means of pulmonary exacerbations (Table 3) and hospitalizations (Table 4) as well as MRC dyspnea scale (Figure 1; Supplementary S2 Table S2) for all QOL-B scales ( $p<0.001$ for each scale).

Table 3. Discrimination of QOL-B scores, stratified by history of pulmonary exacerbations in the previous 12 months (categorized).

\begin{tabular}{lcccc}
\hline \multicolumn{5}{c}{ Mean (SD) QOL-B Scores at Baseline According to Pulmonary Exacerbations } \\
\hline QOL-B Scale & $\mathbf{0}$ & $\mathbf{1 - 2}$ & $\mathbf{2}$ & $\boldsymbol{p}$-Value $\mathbf{~}^{\mathbf{3}}$ \\
\hline Respiratory Symptoms & $62.0(20.7)$ & $56.8(19.9)$ & $49.7(20.7)$ & $<0.001$ \\
Physical Functioning & $47.6(30.5)$ & $44.3(30.1)$ & $33.3(26.5)$ & $<0.001$ \\
Vitality & $46.3(21.8)$ & $43.7(20.9)$ & $36.3(20.1)$ & $<0.001$ \\
Role Functioning & $67.7(25.0)$ & $60.9(26.1)$ & $47.7(27.4)$ & $<0.001$ \\
Health Perceptions & $42.9(24.0)$ & $37.9(21.7)$ & $27.9(19.5)$ & $<0.001$ \\
Emotional Functioning & $73.4(20.3)$ & $71.1(20.0)$ & $63.0(23.7)$ & $<0.001$ \\
Social Functioning & $68.5(23.4)$ & $60.2(25.7)$ & $51.2(28.8)$ & $<0.001$ \\
Treatment Burden & $59.2(24.1)$ & $52.4(24.7)$ & $42.7(24.0)$ & $<0.001$ \\
\hline
\end{tabular}

${ }^{1}$ Differences between groups were assessed by the Kruskal-Wallis test. Abbreviations: QOL-B, Quality of Life Questionnaire-Bronchiectasis; SD, standard deviation.

Table 4. Discrimination of QOL-B scores, stratified by history of prior hospitalization in the previous 12 months.

\begin{tabular}{lccc}
\hline \multicolumn{4}{c}{ Mean (SD) QOL-B Scores at Baseline According to Prior Hospitalization } \\
\hline QOL-B Scale & Yes & No & $p$-Value $\mathbf{1}^{\mathbf{1}}$ \\
\hline Respiratory Symptoms & $51.3(21.3)$ & $59.2(20.3)$ & $<0.001$ \\
Physical Functioning & $32.4(27.3)$ & $47.7(29.8)$ & $<0.001$ \\
Vitality & $39.0(21.5)$ & $43.9(21.1)$ & $<0.001$ \\
Role Functioning & $48.4(27.0)$ & $65.4(25.6)$ & $<0.001$ \\
Health Perceptions & $31.6(21.1)$ & $39.4(22.9)$ & $<0.001$ \\
Emotional Functioning & $66.5(22.9)$ & $70.8(21.1)$ & $<0.001$ \\
Social Functioning & $55.2(27.8)$ & $62.8(25.9)$ & $<0.001$ \\
Treatment Burden & $44.5(24.2)$ & $55.9(24.7)$ & $<0.001$ \\
\hline
\end{tabular}

${ }^{1}$ Differences between groups were assessed by the Mann-Whitney U test. Abbreviations: QOL-B, Quality of Life Questionnaire-Bronchiectasis; SD, standard deviation. 


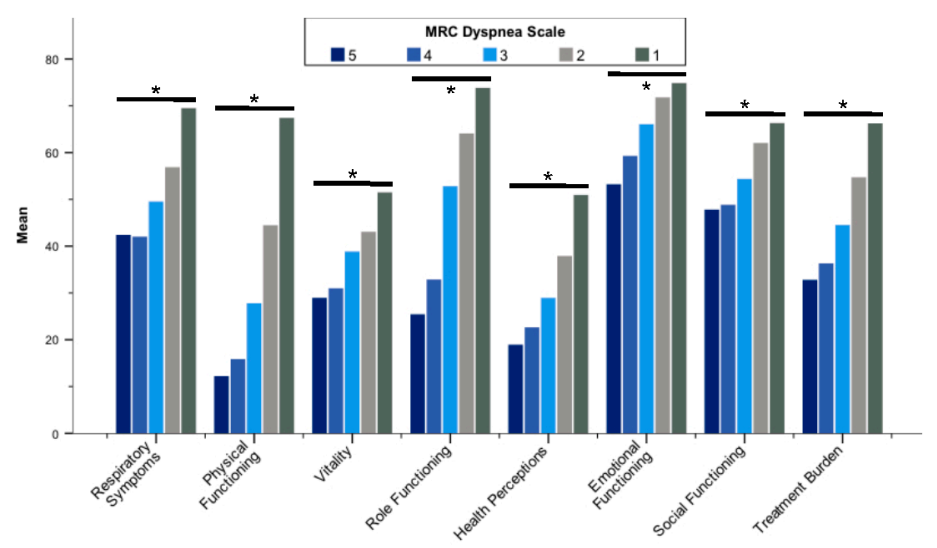

Figure 1. Mean Quality of Life Questionnaire-Bronchiectasis scores stratified by the MRC dyspnea scale. Abbreviation: MRC, Medical Research Council. ${ }^{*} p<0.001$. Differences between groups were assessed by the Kruskal-Wallis test.

All QOL-B scales were worse in patients with more severe bronchiectasis according to BSI categories (Figure 2; Supplementary S2 Table S3). With regard to FEV 1 , QOL-B scales discriminated patients ( $p<0.01$ for each scale), except Social Functioning (Figure 3; Supplementary S2 Table S4). In addition, QoL-B scales were strongly associated with categories of average sputum volume, Pseudomonas aeruginosa infection status and regular pharmacological treatment of bronchiectasis ( $p<0.01$ for each scale), with the exception of Vitality and Emotional Functioning (Figure 4; Supplementary S2 Table S5; Table 5; Supplementary S2 Table S6).

In contrast, only the QOL-B scales Physical Functioning $(p=0.022)$, Role Functioning $(p=0.027)$ and Health Perceptions $(p=0.048)$ were significantly worse in patients with $\geq 3$ lobes affected and/or cystic bronchiectasis, whereas only the Respiratory Symptoms and Social Functioning scales were significantly worse in patients with prior thoracic surgery ( $p=0.025$ and $p=0.002$, respectively; Supplementary S2 Tables S7 and S8).

Moreover, we observed moderate to strong convergence between breathlessness and Respiratory Symptoms, Physical Functioning, Vitality, Role Functioning, Health Perceptions and Treatment Burden ( $r=-0.315$ to $-0.620 ; p<0.001$, each; Table 6). Moderate correlations were found for (a) $\mathrm{FEV}_{1}$ and Respiratory Symptoms, Physical Functioning and Role Functioning ( $r=0.304$ to $0.470 ; p<0.001$, each); (b) pulmonary exacerbation rate and Role Functioning $(r=-0.305, p<0.001)$; (c) hospitalization rate and Role Functioning $(r=-0.339, p<0.001)$; as well as (d) average daily sputum volume and Respiratory Symptoms $(r=-0.321, p<0.001$; Table 6$)$.

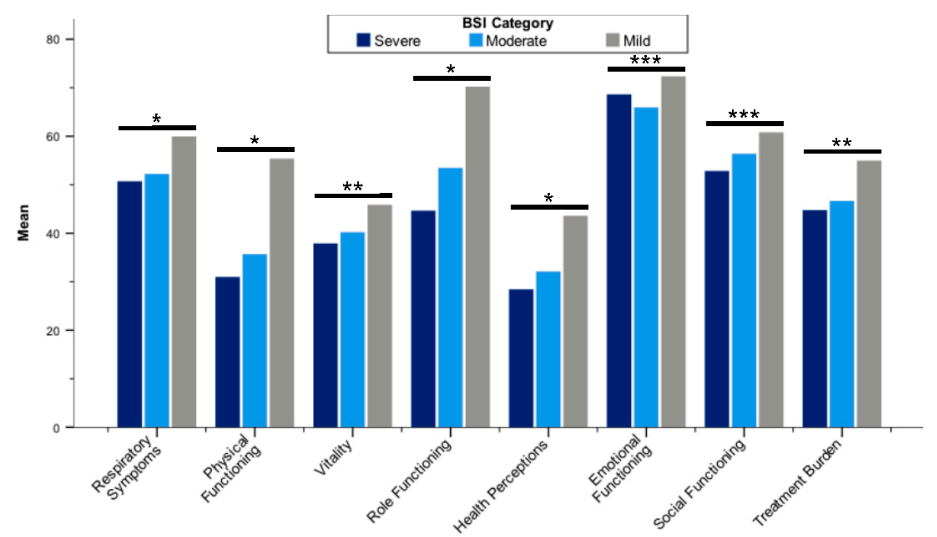

Figure 2. Mean Quality of Life Questionnaire-Bronchiectasis scores stratified by BSI categories. Abbreviation: BSI, Bronchiectasis Severity Index; ${ }^{*} p<0.001 ;{ }^{* *} p<0.01 ;{ }^{* *} p<0.05$. Differences between groups were assessed by the Kruskal-Wallis test. 


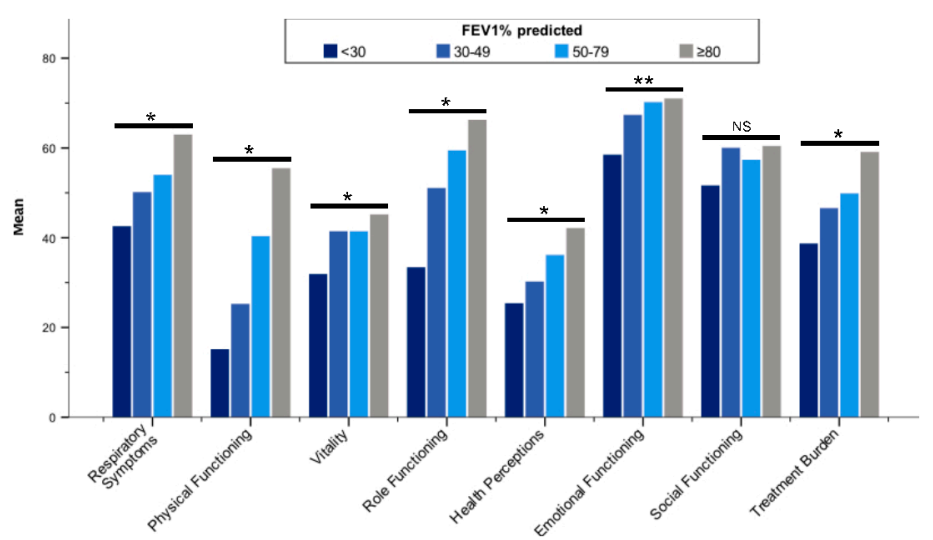

Figure 3. Mean Quality of Life Questionnaire-Bronchiectasis scores stratified by ppFEV 1 (categorized). Abbreviations: $\mathrm{ppFEV}_{1}$, forced expiratory volume in one second (\% predicted); NS, not significant. * $p<0.001 ;{ }^{* *} p<0.01$. Differences between groups were assessed by the Kruskal-Wallis test.

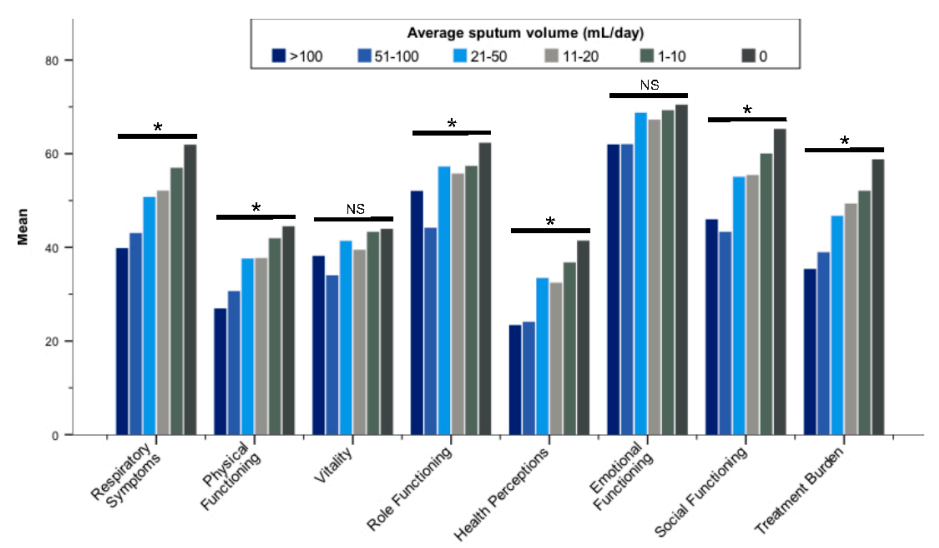

Figure 4. Mean Quality of Life-Bronchiectasis scores stratified by average daily sputum volume (categorized). Abbreviation: NS, not significant. ${ }^{*} p \leq 0.001$; Differences between groups were assessed by the Kruskal-Wallis test.

Table 5. Discrimination of QOL-B scores, stratified by Pseudomonas aeruginosa infection at baseline and/or in the previous 12 months.

\begin{tabular}{lccc}
\hline \multicolumn{4}{c}{ Mean (SD) QOL-B Scores According to Pseudomonas aeruginosa Infection } \\
\hline QOL-B Scale & Yes & No & $p$-Value $\mathbf{1}^{\mathbf{1}}$ \\
\hline Respiratory Symptoms & $51.0(20.1)$ & $59.0(21.0)$ & $<0.001$ \\
Physical Functioning & $35.2(28.2)$ & $45.5(30.0)$ & $<0.001$ \\
Vitality & $40.5(21.4)$ & $42.9(21.3)$ & 0.080 \\
Role Functioning & $52.3(27.4)$ & $62.4(26.7)$ & $<0.001$ \\
Health Perceptions & $31.7(20.3)$ & $38.8(23.4)$ & $<0.001$ \\
Emotional Functioning & $67.2(22.5)$ & $70.2(21.5)$ & 0.063 \\
Social Functioning & $54.5(26.1)$ & $62.8(26.9)$ & $<0.001$ \\
Treatment Burden & $45.8(23.6)$ & $55.0(25.5)$ & $<0.001$ \\
\hline
\end{tabular}

${ }^{1}$ Differences between groups were assessed by the Mann-Whitney U test. Abbreviations: QOL-B, Quality of Life Questionnaire-Bronchiectasis; SD, standard deviation. 
Table 6. Convergent validity: correlation between baseline QOL-B scores and measures of health status.

\begin{tabular}{|c|c|c|c|c|c|}
\hline \multicolumn{6}{|c|}{ Correlations of Baseline Scores on QOL-B Scales with Measures of Health Status } \\
\hline QOL-B Scale & $\mathrm{ppFEV}_{1}$ & $\begin{array}{c}\text { Pulmonary } \\
\text { Exacerbation Rate }\end{array}$ & $\begin{array}{c}\text { Hospitalization } \\
\text { Rate }\end{array}$ & $\begin{array}{c}\text { MRC Dyspnea } \\
\text { Scale }\end{array}$ & $\begin{array}{l}\text { Average Sputum } \\
\text { Volume (mL/Day) }\end{array}$ \\
\hline Respiratory Symptoms & $r=0.304$ & $r=-0.241$ & $r=-0.223$ & $r=-0.414$ & $r=-0.321$ \\
\hline$(n=892)$ & $p<0.001$ & $p<0.001$ & $p<0.001$ & $p<0.001$ & $p<0.001$ \\
\hline Physical Functioning & $r=0.470$ & $r=-0.203$ & $r=-0.282$ & $r=-0.620$ & $r=-0.139$ \\
\hline$(n=889)$ & $p<0.001$ & $p<0.001$ & $p<0.001$ & $p<0.001$ & $p<0.001$ \\
\hline Vitality & $r=0.152$ & $r=-0.198$ & $r=-0.139$ & $r=-0.315$ & $r=-0.088$ \\
\hline$(n=892)$ & $p<0.001$ & $p<0.001$ & $p<0.001$ & $p<0.001$ & $p=0.009$ \\
\hline Role Functioning & $r=0.334$ & $r=-0.305$ & $r=-0.339$ & $r=-0.513$ & $r=-0.163$ \\
\hline$(n=898)$ & $p<0.001$ & $p<0.001$ & $p<0.001$ & $p<0.001$ & $p<0.001$ \\
\hline Health Perceptions & $r=0.256$ & $r=-0.263$ & $r=-0.198$ & $r=-0.417$ & $r=-0.215$ \\
\hline$(n=891)$ & $p<0.001$ & $p<0.001$ & $p<0.001$ & $p<0.001$ & $p<0.001$ \\
\hline Emotional Functioning & $r=0.107$ & $r=-0.176$ & $r=-0.108$ & $r=-0.239$ & $r=-0.090$ \\
\hline$(n=889)$ & $p=0.003$ & $p<0.001$ & $p=0.001$ & $p<0.001$ & $p=0.008$ \\
\hline Social Functioning & $r=0.087$ & $r=-0.240$ & $r=-0.162$ & $r=-0.225$ & $r=-0.236$ \\
\hline$(n=878)$ & $p=0.011$ & $p<0.001$ & $p<0.001$ & $p<0.001$ & $p<0.001$ \\
\hline Treatment Burden & $r=0.229$ & $r=-0.260$ & $r=-0.236$ & $r=-0.370$ & $r=-0.239$ \\
\hline$(n=645)$ & $p<0.001$ & $p<0.001$ & $p<0.001$ & $p<0.001$ & $p<0.001$ \\
\hline
\end{tabular}
$r<0.3$ weak correlation $r=0.3-0.49$ moderate correlation $r>0.5$ strong correlation

Convergent validity is shown as heat map of $r$-values. $p$-values were assessed by the Spearman's rank correlation coefficient $(r)$. Abbreviations: $\mathrm{ppFEV}_{1}$, forced expiratory volume in $1 \mathrm{~s}(\%$ predicted); MRC, Medical Research Council; QOL-B, Quality of Life Questionnaire-Bronchiectasis.

\subsection{Minimal Clinical Important Difference}

The derived MCIDs ranged between 8.5 points for the Respiratory Symptoms scale and 14.1 points for the Social Functioning scale (Table 7).

Table 7. MCID estimates for the QOL-B scales.

\begin{tabular}{lc}
\hline QOL-B Scale & MCID \\
\hline Respiratory Symptoms & 8.5 \\
Physical Functioning & 8.7 \\
Vitality & 10.5 \\
Role Functioning & 10.4 \\
Health Perceptions & 10.3 \\
Emotional Functioning & 9.3 \\
Social Functioning & 14.1 \\
Treatment Burden & 13.0 \\
\hline Abbreviations: MCID, minimal clinical important difference; QOL-B, Quality of Life Questionnaire-Bronchiectasis.
\end{tabular}

\section{Discussion}

The present study confirms that the German translation of the QOL-B, version 3.1, is a valid tool for the assessment of disease-specific QOL among adult patients with bronchiectasis. We showed that it is internally consistent, reproducible over a 2-week period and has good construct validity, with most scales discriminating between patients based on symptom burden as well as established measures of disease severity of bronchiectasis.

Our findings are comparable to those of the original QOL-B validation study by Quittner and colleagues [12] as well as a validation study of the QOL-B, version 3.0, by Olveira and colleagues [18]. Overall, we observed only small floor or ceiling effects, which is a prerequisite for the detection of future changes [10]. While slightly more patients had a QOL-B score of " 0 " compared to the studies by Quittner et al. and Olveira et al. (maximum $10.7 \%$ vs. $5.1 \%$ and $3.4 \%$, respectively), scores of " 100 " were less frequently observed in our study (maximum $10.9 \%$ vs. $24.1 \%$ and $21.7 \%$, respectively) $[12,18]$. We 
demonstrated good to excellent internal consistency, with Cronbach's $\alpha$ ranging from 0.73 to 0.92 , which is accordance with the results of the two previous studies (Cronbach's $\alpha$ 0.70-0.91, each) [12,18]. Similarly, we found good to excellent test-retest reliability in line with the above-mentioned studies (ICCs $0.84-0.96$ vs. 0.72-0.86 and 0.68-0.88, respectively) [12,18], thus supporting the appropriateness of our reproducibility analysis, even if clearly less datasets were available for assessment. Moreover, our MCID estimates of QOL-B scales showed a range of 8.5-14.1 points, with 8.5 points for the Respiratory Symptoms scale. This is in agreement with the MCID ranges of 7.7-12.6 and 8.2-13.3 points, with a MCID of 7.7 and 8.2 points for the Respiratory Symptoms scale in the AIR-BX1 and AIR-BX2 studies, respectively [12], when determined by the SEM method. Similarly, in the Spanish validation study the MCID was 6.8 points for the Respiratory Symptoms scale [18]. In order to establish discriminant and convergent validity we analyzed several established measures of health status and disease severity and found good discrimination as well as moderate to strong correlations with the majority of the QOL-B scales, with the exception of the Vitality, Emotional Functioning and Social Functioning scales.

However, we also found some differences when comparing our results to those of previous studies on the QOL-B's validity. In our study, all QOL-B scales except Social Functioning discriminated very well between patients based on $\mathrm{FEV}_{1}$, with moderate correlations found between Respiratory Symptoms, Physical Functioning as well as Role Functioning scales and $\mathrm{FEV}_{1}$. In this regard, the respective results by Quittner et al. were less strong in the setting of two RCTs, with merely moderate correlation found between Physical Functioning and $\mathrm{FEV}_{1}$ in the AIR-BX2 trial [12]. Furthermore, we observed that the Treatment Burden scale was generally worse in patients with more severe bronchiectasis and higher symptom burden. This is in contrast to the study by Olveira et al., who found that the Treatment Burden scale discriminated only between patients according to radiological severity, as assessed by the Bhalla score [18]. In this regard, one should keep in mind that in our study 259 subjects (28.7\%) skipped the Treatment Burden scale, as they were not receiving bronchiectasis treatment, potentially limiting its relevance to the general bronchiectasis population.

The existing QOL questionnaires, which have been used in bronchiectasis studies, have advantages and disadvantages. The SGRQ has been most widely applied. While it has good psychometric properties, it consists of 50 items, thus making it rather complex $[8,16]$. Moreover, it has primarily been developed for use in other chronic respiratory conditions, with a greater focus on breathlessness, and has never obtained broad acceptance for routine use in Germany. In this regard, the lower respiratory tract infections-visual analogue scale (LRTI-VAS) [23], the COPD Assessment Test (CAT) [24] and the Leicester Cough Questionnaire (LCQ) [25] are more feasible, but are neither specific for use in bronchiectasis, with the later mainly focusing on health related to cough.

The QOL-B was the first questionnaire specifically developed for the assessment of QOL among patients with bronchiectasis [12,21]. It has 37 items resulting in 8 scales, with no overall score provided. Data on the responsiveness of the Respiratory Symptoms scale from RCTs are conflicting [16]. While the QOL-B Respiratory Symptoms scale significantly improved along with reductions of bacterial load during on-treatment periods in the RCTs ORBIT-3 and -4 [26], it turned out to be unresponsive in several other RCTs despite evidence for other clinical meaningful changes [27-29]. However, this observation has previously been made for the SGRQ and the LCQ [29-31], indicating that, amongst others, responsiveness may depend on differences in patient populations and the type of intervention $[14,16]$.

Therefore, other disease-specific QOL questionnaires have been proposed as alternatives to the QOL-B $[14,15]$. The Bronchiectasis Health Questionnaire (BHQ) is brief and simple-to-use. Its 10 items result in a single, one-dimensional overall score [14]. While psychometric data supporting its use are available, its responsiveness and MCID have not been studied in detail yet [16]. Very recently, the Bronchiectasis Impact Measure (BIM) has been developed and validated as a simple PROM [15]. In contrast to other questionnaires, 
it is composed of 8 visual analogue scales representing unique domains and focuses on the impact of bronchiectasis symptoms on patients' lives. This appears to be particularly useful with ambiguous symptoms, which are typically perceived by patients with considerable interindividual variability, such as sputum, with some patients regarding increased sputum production as positive, while others think of it as negative [8]. Notably, this allowed the authors of the BIM to provide patient-derived MCID estimates [15]. While the BIM was strongly to very strongly correlated with QOL-B scores, it appeared to outperform the QOL-B by means of convergent validity (and the degree of floor and ceiling effects), with most of its scales showing moderate to very strong correlation with measures of disease severity [15]. However, direct comparison between both measures of disease-specific QOL from multicenter studies is lacking. A recent comprehensive systematic review and meta-analysis of health-related QOL questionnaires in bronchiectasis concludes that good psychometric data on the bronchiectasis-specific QOL questionnaires are emerging, but further studies on their medium- to long-term test-retest reliability, responsiveness and MCID are required. Currently, only the CAT, the QOL-B and the BIM have provided MCID values for bronchiectasis populations [16].

Our study has strengths and limitations. Most importantly, the data underlying our study were captured at 38 sites from all levels of the German healthcare system with the broadest possible geographical distribution, including not only secondary and tertiary care centers but also practitioners in private practice, and may therefore be regarded both nationally representative and related to real life. As a consequence, we were able to include a large number of patients as well as a multitude of measure of health status and disease severity in our analysis. However, the key limitation is that we were unable to evaluate responsiveness due to our cross-sectional study design. In this regard, we will utilize the registry data as fully validated follow-up datasets will become available in the near future. Another limitation is that we used a distribution-based method of estimating MCIDs only (SEM method). In contrast to anchor-based or patient-derived MCIDs, which may be more clinically relevant as they evaluate the direct impact on the patients, our MCIDs are mathematically derived and essentially measure the variances of scores [15]. However, there is no single agreed method of estimating the MCID for PROMs and commonly accepted anchors are unavailable for most of the bronchiectasisassociated symptoms [15,32]. Lastly, we decided not to include additional questionnaires as further PROMs for comparison to QOL-B scores, as provided by other studies $[12,15,18,23]$, considering feasibility of data collection within our registry. Therefore, we are unable to draw conclusions about the superiority of a particular tool for the assessment of QOL in patients with bronchiectasis in our setting.

Even though our findings may be largely confirmative, our study is of importance as until now there was no validated tool ready for use among the German bronchiectasis population. The epidemiology of bronchiectasis and the standard of care, keeping the absence of any licensed pharmacological treatment in mind, show distinct differences between geographical regions and healthcare systems, thus supporting the need for studies in different bronchiectasis populations and justifying the present analysis [33]. QOL is not only an important PROM in RCTs but also matters to patients in their everyday lives [9]. Our findings provide evidence that the QOL-B is a valid tool and offer guidance for its interpretation in clinical practice. In this respect, our study may contribute to the recognition of disease-specific QOL as a crucial criterion for clinical decision-making in the routine management of bronchiectasis in Germany.

\section{Conclusions}

We validated the German translation of the QOL-B, version 3.1, in a representative cohort of adult German patients with bronchiectasis. However, its responsiveness needs to be established during registry follow-up by means of changes in health status, including clinical deterioration due to pulmonary exacerbations as well as improvement due to continuous specialized care and targeted therapeutic interventions. 
Supplementary Materials: The following are available online at https:/ /www.mdpi.com/article/10 $.3390 / j \mathrm{jm} 11020441 / \mathrm{s} 1$, Supplementary file S1: SAS and SPSS Program Codes for Scoring the QOL-B Version 3.1 and Manual Scoring Instructions for QOL-B Version 3.1. Furthermore, Supplementary S2 Table S1. Comparison of demographic and baseline characteristics between all subjects enrolled in PROGNOSIS until Mach $2018(n=1000)$ and those who completed the QOL-B $(n=904)$; Supplementary S2 Table S2. Discrimination of QOL-B scores, stratified by MRC dyspnea scale; Supplementary S2 Table S3. Discrimination of QOL-B scores, stratified by BSI categories $(n=666)$; Supplementary S2 Table S4. Discrimination of QOL-B scores, stratified by ppFEV 1 (categorized); Supplementary S2 Table S5. Discrimination of QOL-B scores, stratified by average daily sputum volume (categorized); Supplementary S2 Table S6. Discrimination of QOL-B scores, stratified by regular pharmacological treatment of bronchiectasis; Supplementary S2 Table S7. Discrimination of QOL-B scores, stratified by radiological severity; Supplementary S2 Table S8. Discrimination of QOL-B scores, stratified by history of prior thoracic surgery.

Author Contributions: Conceptualization, G.B.-N., A.d.R., R.D., P.M., I.P., J.R., A.Z. and F.C.R.; methodology, L.Q. and F.C.R.; validation, L.Q., G.B.-N., I.P., J.R., L.Q. and F.C.R.; formal analysis, L.Q. and F.C.R.; investigation, A.d.R., I.P., P.M., J.R., S.S., F.C.R. and PROGNOSIS study group; resources, G.B.-N., A.d.R., P.M., T.W., A.Z. and F.C.R.; data curation, G.B.-N. and F.C.R.; writing-original draft preparation, L.Q. and F.C.R.; writing-review and editing, L.Q., G.B.-N., A.d.R., R.D., P.M., I.P., J.R., S.S., T.W., A.Z. and F.C.R.; visualization, L.Q. and F.C.R.; supervision, A.d.R., P.M., J.R. and F.C.R.; project administration, G.B.-N., A.Z. and F.C.R.; funding acquisition, P.M., J.R., T.W., A.Z. and F.C.R. All authors have read and agreed to the published version of the manuscript.

Funding: The German bronchiectasis registry PROGNOSIS is financially supported by Bayer Vital, Grifols, Insmed Inc., InfectoPharm, the German Ministry of Research and Education (BMBF) via the German Center for Lung Research (DZL) and the German Center for Infection Research (DZIF) as well as the European bronchiectasis registry EMBARC via the Innovative Medicines Initiative (IMI) and EFPIA companies under the European Commission funded project, iABC (grant 115721). The funders had no role in the design of the study; in the collection, analyses, or interpretation of data; in the writing of the manuscript, or in the decision to publish the results.

Institutional Review Board Statement: The study was conducted according to the guidelines of the Declaration of Helsinki and approved by the Institutional Review Boards (or Ethics Committees) of all participating centers, referring to the initial ethical approval of Hannover Medical School (MHH; No. 6656/2015).

Informed Consent Statement: Informed consent was obtained from all subjects involved in the study.

Data Availability Statement: Data are available from the authors upon request.

Acknowledgments: Members of the PROGNOSIS study group: B. Grün, Bad Windsheim; S. Dargel and K. Ludwig, Baunatal; A. de Roux, Berlin; R. Otto-Knapp, Berlin; H. Lode, Berlin; C. Gogoll, Berlin; M. Probst, Berlin; F. Herrmann, Bielefeld; A. Overlack and S. Pabst, Bonn; U. Sommerwerck, Braunschweig, Wuppertal and Köln; H. Vehar, Buchholz; S. Blaas, Donaustauf; B. Schaaf, Dortmund; M. Kolditz, Dresden; S. Sutharsan, Essen; P. Kardos, Frankfurt/Main; A. Grünewaldt, Frankfurt; S. Sorichter and T. Scholz, Freiburg; M. Idzko and M. Mohadjer, Freiburg; S. Eisenmann, Halle; S.P. Aries and J. Lauer-Hermfisse, Hamburg; S. Kampf, Hamburg; F.C. Ringshausen, Hannover; S. Wege and F. Herth, Heidelberg; S. Ewig, Herne; C. Reinhardt and S. Andreas, Immenhausen; C. Schumann, Immenstadt; I. Bobis and T. Bahmer, Kiel; R. Fey, Koblenz; M. Jüch, Lostau; A.T. Kempa, Löwenstein; E. Piirsoo and B. Klapdor, Marburg; P. Mertsch, München; B. Schmidt, Münster; H. Hein, Reinbek; P. Haidl, Schmallenberg; and J.F. Gamarra, Straubing. The authors and investigators would like to thank the participating patients; A. Quittner for providing the German translation of the QOL-B, version 3.1; J.D. Chalmers and the members of the EMBARC Steering Committee for friendship, enthusiasm and support; as well as CAPNETZ STIFTUNG for project support with regard to study implementation and administration, data management as well as database maintenance, in particular W. Kröner, J. Naim and M.-L. Witte. L.Q., J.R., T.W., A.Z. and F.C.R. are grateful to P. Sokol, B. Staar and R. Zimmer for data entry and to $S$. Konwert for her support with data management and data cleaning as well as the team of the DZL partner site BREATH for administrative and scientific support, in particular I. Kwapniewska, A. Barth, E. Missol-Kolka and I. Schäfer. Preliminary data of our study were presented at the ERS International Congress 2018 and the annual congress of the German Respiratory Society in 
2019 , using a provisional dataset for analysis. L.Q. conducted this work as part of her MD thesis. She thanks J. Fuge for serving as co-supervisor of her thesis.

Conflicts of Interest: L.Q., G.B.N., A.D.R. and A.Z. declare no conflicts of interest. R.D. and/or his institution received grants, advisory/lecture/clinical trial fees and non-financial support by Bayer, Cepheid, DiaSorin, Insmed, Oxford Immunotec, Qiagen, Quidel, BMBF, DZL and DZIF. P.M. and/or his institution received grants, advisory/lecture/clinical trial fees and non-financial support by AstraZeneca, Boehringer Ingelheim, Insmed Inc., Vertex and DZL. I.P. and/or her institution received grants, advisory/lecture/clinical trial fees and non-financial support from Chiesi and Förderstiftung $\mathrm{MHH}^{\text {Plus }}$. J.R. and/or her institution received grants, advisory/lecture/clinical trial fees and nonfinancial support from AstraZeneca, Bayer, Brahms, Chiesi, Esanum, Grifols, GSK, Novartis, DZL, DZIF and BMBF. S.S. received personal fees or grants from Boehringer Ingelheim, Celtaxsys, Chiesi, Corbus Pharmaceuticals Flatley, Galapagos, Insmed Inc., Ionis Pharmaceuticals, Novartis, Proteostasis Therapeutics, Teva and Vertex outside the submitted work. S.S. has served as an investigator in clinical trials for AstraZeneca, Zambon, Insmed Inc., Novartis and Vertex. T.W. received grants to his institution from Insmed Inc. and Novartis and personal fees for advisory boards and lectures by AstraZeneca, Insmed Inc., Novartis and Zambon. F.C.R. and/or his institution received grants, advisory/lecture/clinical trial fees and non-financial support by AstraZeneca, Bayer, Boehringer Ingelheim, Celtaxsys, Chiesi, Corbus, Grifols, InfectoPharm, Insmed Inc., Novartis, PARI, Parion, Polyphor, Shionogi, Vertex, Zambon, DZL, DZIF, BMBF and IMI/EFPIA.

\section{References}

1. Aliberti, S.; Goeminne, P.C.; O’Donnell, A.E.; Aksamit, T.R.; Al-Jahdali, H.; Barker, A.F.; Blasi, F.; Boersma, W.G.; Crichton, M.L.; De Soyza, A.; et al. Criteria and definitions for the radiological and clinical diagnosis of bronchiectasis in adults for use in clinical trials: International consensus recommendations. Lancet Respir. Med. 2021, Online ahead of print.

2. Quint, J.K.; Millett, E.R.; Joshi, M.; Navaratnam, V.; Thomas, S.L.; Hurst, J.R.; Smeeth, L.; Brown, J.S. Changes in the incidence, prevalence and mortality of bronchiectasis in the UK from 2004 to 2013: A population-based cohort study. Eur. Respir. J. 2016, 47, 186-193. [CrossRef] [PubMed]

3. Ringshausen, F.C.; Rademacher, J.; Pink, I.; de Roux, A.; Hickstein, L.; Ploner, T.; Welte, T.; Diel, R. Increasing bronchiectasis prevalence in Germany, 2009-2017: A population-based cohort study. Eur. Respir. J. 2019, 54, 1900499. [CrossRef] [PubMed]

4. Diel, R.; Chalmers, J.D.; Rabe, K.F.; Nienhaus, A.; Loddenkemper, R.; Ringshausen, F.C. Economic burden of bronchiectasis in germany. Eur. Respir. J. 2019, 53, 1802033. [CrossRef]

5. Loebinger, M.R.; Polverino, E.; Chalmers, J.D.; Tiddens, H.; Goossens, H.; Tunney, M.; Ringshausen, F.C.; Hill, A.T.; Pathan, R.; Angyalosi, G.; et al. Efficacy and safety of TOBI podhaler in pseudomonas aeruginosa-infected bronchiectasis patients: iBEST study. Eur. Respir. J. 2021, 57, 2001451. [CrossRef]

6. Haworth, C.S.; Bilton, D.; Chalmers, J.D.; Davis, A.M.; Froehlich, J.; Gonda, I.; Thompson, B.; Wanner, A.; O’Donnell, A.E. Inhaled liposomal ciprofloxacin in patients with non-cystic fibrosis bronchiectasis and chronic lung infection with pseudomonas aeruginosa (ORBIT-3 and ORBIT-4): Two phase 3, randomised controlled trials. Lancet Respir. Med. 2019, 7, 213-226. [CrossRef]

7. Barker, A.F.; O’Donnell, A.E.; Flume, P.; Thompson, P.J.; Ruzi, J.D.; de Gracia, J.; Boersma, W.G.; De Soyza, A.; Shao, L.; Zhang, J.; et al. Aztreonam for inhalation solution in patients with non-cystic fibrosis bronchiectasis (AIR-BX1 and AIR-BX2): Two randomised double-blind, placebo-controlled phase 3 trials. Lancet Respir. Med. 2014, 2, 738-749. [CrossRef]

8. Bilton, D.; Daviskas, E.; Anderson, S.D.; Kolbe, J.; King, G.; Stirling, R.G.; Thompson, B.R.; Milne, D.; Charlton, B. Phase 3 randomized study of the efficacy and safety of inhaled dry powder mannitol for the symptomatic treatment of non-cystic fibrosis bronchiectasis. Chest 2013, 144, 215-225. [CrossRef]

9. Aliberti, S.; Masefield, S.; Polverino, E.; De Soyza, A.; Loebinger, M.R.; Menendez, R.; Ringshausen, F.C.; Vendrell, M.; Powell, P.; Chalmers, J.D.; et al. Research priorities in bronchiectasis: A consensus statement from the EMBARC clinical research collaboration. Eur. Respir. J. 2016, 48, 632-647. [CrossRef]

10. U.S. Food \& Drug Administration (FDA). Patient-Reported Outcome Measures: Use in Medical Product Development to Support Labeling Claims. 2009. Available online: https://www.fda.gov/regulatory-information/search-fda-guidance-documents/ patient-reported-outcome-measures-use-medical-product-development-support-labeling-claims (accessed on 20 November 2021).

11. Flume, P.A.; Griffith, D.E.; Chalmers, J.D.; Daley, C.L.; Olivier, K.; O’Donnell, A.; Aksamit, T.; Kasperbauer, S.; Leitman, A.; Winthrop, K.L. Development of drugs for nontuberculous mycobacterial disease: Clinicians' interpretation of a US Food and Drug Administration workshop. Chest 2021, 159, 537-543. [CrossRef]

12. Quittner, A.L.; O’Donnell, A.E.; Salathe, M.A.; Lewis, S.A.; Li, X.; Montgomery, A.B.; O'Riordan, T.G.; Barker, A.F. Quality of life questionnaire-bronchiectasis: Final psychometric analyses and determination of minimal important difference scores. Thorax 2015, 70, 12-20. [CrossRef] 
13. Spinou, A.; Fragkos, K.C.; Lee, K.K.; Elston, C.; Siegert, R.J.; Loebinger, M.R.; Wilson, R.; Garrod, R.; Birring, S.S. The validity of health-related quality of life questionnaires in bronchiectasis: A systematic review and meta-analysis. Thorax 2016, 71, 683-694. [CrossRef]

14. Spinou, A.; Siegert, R.J.; Guan, W.J.; Patel, A.S.; Gosker, H.R.; Lee, K.K.; Elston, C.; Loebinger, M.R.; Wilson, R.; Garrod, R.; et al. The development and validation of the Bronchiectasis Health Questionnaire. Eur. Respir. J. 2017, 49, 1601532. [CrossRef]

15. Crichton, M.L.; Dudgeon, E.K.; Shoemark, A.; Chalmers, J.D. Validation of the Bronchiectasis Impact Measure (BIM): A novel patient-reported outcome measure. Eur. Respir. J. 2021, 57, 2003156. [CrossRef]

16. McLeese, R.H.; Spinou, A.; Alfahl, Z.; Tsagris, M.; Elborn, J.S.; Chalmers, J.D.; De Soyza, A.; Loebinger, M.R.; Birring, S.S.; Fragkos, K.C.; et al. Psychometrics of health-related quality of life questionnaires in bronchiectasis: A systematic review and meta-analysis. Eur. Respir. J. 2021, 58, 2100025. [CrossRef]

17. Chalmers, J.D.; Goeminne, P.; Aliberti, S.; McDonnell, M.J.; Lonni, S.; Davidson, J.; Poppelwell, L.; Salih, W.; Pesci, A.; Dupont, L.J.; et al. The Bronchiectasis Severity Index. An international derivation and validation study. Am. J. Respir. Crit. Care Med. 2014, 189, 576-585. [CrossRef]

18. Olveira, C.; Olveira, G.; Espildora, F.; Giron, R.M.; Munoz, G.; Quittner, A.L.; Martinez-Garcia, M.A. Validation of a quality of life questionnaire for bronchiectasis: Psychometric analyses of the Spanish QOL-B-V3.0. Qual. Life Res. 2014, 23, 1279-1292. [CrossRef] [PubMed]

19. Rademacher, J.; de Roux, A.; Ringshausen, F.C. [PROGNOSIS-The prospective German non-cf bronchiectasis patient registry]. Pneumologie 2015, 69, 391-393. [PubMed]

20. Reiff, D.B.; Wells, A.U.; Carr, D.H.; Cole, P.J.; Hansell, D.M. CT findings in bronchiectasis: Limited value in distinguishing between idiopathic and specific types. AJR Am. J. Roentgenol. 1995, 165, 261-267. [CrossRef] [PubMed]

21. Quittner, A.L.; Marciel, K.K.; Salathe, M.A.; O’Donnell, A.E.; Gotfried, M.H.; Ilowite, J.S.; Metersky, M.L.; Flume, P.A.; Lewis, S.A.; McKevitt, M.; et al. A preliminary quality of life questionnaire-bronchiectasis: A patient-reported outcome measure for bronchiectasis. Chest 2014, 146, 437-448. [CrossRef]

22. Field, A. Correlation. In Discovering Statistics Using SPSS, 3rd ed.; SAGE Publications Ltd.: London, UK, 2009 ; pp. 166-196.

23. Altenburg, J.; Wortel, K.; de Graaff, C.S.; van der Werf, T.S.; Boersma, W.G. Validation of a visual analogue score (LRTI-VAS) in non-CF bronchiectasis. Clin. Respir. J. 2016, 10, 168-175. [CrossRef] [PubMed]

24. Finch, S.; Laska, I.F.; Abo-Leyah, H.; Fardon, T.C.; Chalmers, J.D. Validation of the COPD Assessment Test (CAT) as an outcome measure in bronchiectasis. Chest 2020, 157, 815-823. [CrossRef]

25. Murray, M.P.; Turnbull, K.; MacQuarrie, S.; Pentland, J.L.; Hill, A.T. Validation of the Leicester Cough Questionnaire in non-cystic fibrosis bronchiectasis. Eur. Respir. J. 2009, 34, 125-131. [CrossRef]

26. Chalmers, J.D.; Cipolla, D.; Thompson, B.; Davis, A.M.; O’Donnell, A.; Tino, G.; Gonda, I.; Haworth, C.; Froehlich, J. Changes in respiratory symptoms during 48-week treatment with ARD-3150 (inhaled liposomal ciprofloxacin) in bronchiectasis: Results from the ORBIT-3 and -4 studies. Eur. Respir. J. 2020, 56, 2000110. [CrossRef] [PubMed]

27. Crichton, M.L.; Lonergan, M.; Barker, A.F.; Sibila, O.; Goeminne, P.; Shoemark, A.; Chalmers, J.D. Inhaled aztreonam improves symptoms of cough and sputum production in patients with bronchiectasis: A post hoc analysis of the AIR-BX studies. Eur. Respir. J. 2020, 56, 2000608. [CrossRef] [PubMed]

28. Aksamit, T.; De Soyza, A.; Bandel, T.J.; Criollo, M.; Elborn, J.S.; Operschall, E.; Polverino, E.; Roth, K.; Winthrop, K.L.; Wilson, R. RESPIRE 2: A phase III placebo-controlled randomised trial of ciprofloxacin dry powder for inhalation in non-cystic fibrosis bronchiectasis. Eur. Respir. J. 2018, 51, 1702053. [CrossRef] [PubMed]

29. De Soyza, A.; Aksamit, T.; Bandel, T.J.; Criollo, M.; Elborn, J.S.; Operschall, E.; Polverino, E.; Roth, K.; Winthrop, K.L.; Wilson, R. RESPIRE 1: A phase III placebo-controlled randomised trial of ciprofloxacin dry powder for inhalation in non-cystic fibrosis bronchiectasis. Eur. Respir. J. 2018, 51, 1702052. [CrossRef] [PubMed]

30. Serisier, D.J.; Bilton, D.; De Soyza, A.; Thompson, P.J.; Kolbe, J.; Greville, H.W.; Cipolla, D.; Bruinenberg, P.; Gonda, I. Inhaled, dual release liposomal ciprofloxacin in non-cystic fibrosis bronchiectasis (ORBIT-2): A randomised, double-blind, placebo-controlled trial. Thorax 2013, 68, 812-817. [CrossRef] [PubMed]

31. Serisier, D.J.; Martin, M.L.; McGuckin, M.A.; Lourie, R.; Chen, A.C.; Brain, B.; Biga, S.; Schlebusch, S.; Dash, P.; Bowler, S.D. Effect of long-term, low-dose erythromycin on pulmonary exacerbations among patients with non-cystic fibrosis bronchiectasis: The BLESS randomized controlled trial. JAMA 2013, 309, 1260-1267. [CrossRef] [PubMed]

32. Revicki, D.; Hays, R.D.; Cella, D.; Sloan, J. Recommended methods for determining responsiveness and minimally important differences for patient-reported outcomes. J. Clin. Epidemiol. 2008, 61, 102-109. [CrossRef]

33. Dhar, R.; Singh, S.; Talwar, D.; Mohan, M.; Tripathi, S.K.; Swarnakar, R.; Trivedi, S.; Rajagopala, S.; D'Souza, G.; Padmanabhan, A.; et al. Bronchiectasis in India: Results from the European Multicentre Bronchiectasis Audit and Research Collaboration (EMBARC) and Respiratory Research Network of India Registry. Lancet Glob. Health 2019, 7, e1269-e1279. [CrossRef] 